\title{
BMJ Open How much do we know about the effectiveness of warm-up intervention on work related musculoskeletal disorders, physical and psychosocial functions: protocol for a systematic review
}

\author{
Nicolas Larinier, ${ }^{1,2}$ Romain Balaguier (1) , ${ }^{1,2}$ Nicolas Vuillerme ${ }^{1,2,3}$
}

To cite: Larinier N, Balaguier $\mathrm{R}$, Vuillerme N. How much do we know about the effectiveness of warm-up intervention on work related musculoskeletal disorders, physical and psychosocial functions: protocol for a systematic review. BMJ Open 2020;10:e039063. doi:10.1136/ bmjopen-2020-039063

- Prepublication history and additional materials for this paper is available online. To view these files, please visit the journal online (http://dx.doi. org/10.1136/bmjopen-2020039063).

Received 03 April 2020 Revised 12 0ctober 2020 Accepted 25 October 2020

Check for updates

(C) Author(s) (or their employer(s)) 2020. Re-use permitted under CC BY-NC. No commercial re-use. See rights and permissions. Published by BMJ.

${ }^{1}$ AGEIS, University Grenoble Alpes, Grenoble, France

${ }^{2}$ Opti'Mouv, St Paul, France ${ }^{3}$ Institut Universitaire de France, Paris, France

Correspondence to Dr Romain Balaguier; romain.balaguier@hotmail.fr

\section{ABSTRACT}

Introduction Work-related musculoskeletal disorders (WMSDs) are a growing worldwide burden and effective interventions to prevent them are needed. Physical activity at the workplace is now recognised as a relevant component of WMSDs prevention. Along these lines, warm-up interventions are now offered in a large number of companies to manage WMSDs. Although benefits of warm-up have been previously documented in sports context, to the best of our knowledge, the effectiveness of such intervention in workplaces still remains to be established. Within this context, the aim of the present review is to identify from published literature the available evidence regarding the effects of warm-up on WMSDs and physical and psychosocial functions.

Methods The following electronic databases will be searched (from inception onwards to June 2020): Cochrane Central Register of Controlled Trials, PubMed (Medline), Web of Science and Physiotherapy Evidence Database. Randomised and non-randomised controlled studies will be included in this review. Participants should be adult employees without specific comorbidities. Interventions should include a warm-up physical intervention in realworkplaces. The primary outcomes will be pain, discomfort or fatigue. The secondary outcomes will be job control or motivation at work. This review will follow the Preferred Reporting Items for Systematic Reviews and Meta-Analyses guidelines and two team members will independently screen all citations, full-text articles and abstract data. A systematic narrative synthesis will be provided with information presented in the text and tables to summarise the characteristics and findings of the included studies. Ethics and dissemination The approval of an ethical committee is not required. All the included studies will comply with the current ethical standards. The results of this review will summarise the effects of warm-up intervention on WMSDs, physical or psychosocial functions. This information could help professionals in decision making related to the use of these interventions to prevent WMSDs. Findings will be disseminated to academic audiences through peerreviewed publications, as well as to policy-makers. PROSPERO registration number CRD42019137211.
Strengths and limitations of this study

- This study will be to the best of our knowledge the first review to critically appraise the effectiveness of warm-up exercises to prevent work-related musculoskeletal disorders in workplaces.

- Reporting in accordance with the Preferred Reporting Items for Systematic Reviews and Meta Analyses statement.

- This study will include both randomised controlled trial (RCT) and non-RCT.

- A low number of studies and significant heterogeneity is expected that might prevent performing a meta-analysis of the results.

\section{INTRODUCTION}

Work-related musculoskeletal disorders (WMSDs) are conditions affecting muscles, tendons, nerves, ligaments, joints or spinal discs. ${ }^{1}$ They are now considered as a public health problem all over the world since their adverse consequences on quality of life and work participation are important. ${ }^{2-6}$ This underlines the importance of finding effective prevention or curative strategies/interventions. In the last two decades, numerous researchers have identified the workplace as an ideal setting to support the promotion of healthier lifestyle and to prevent WMSDs. ${ }^{7-9}$ Hence, the use of workplace physical activity interventions for the management of WMSDs is now well supported by scientific evidence. ${ }^{10-16}$ Interestingly, WMSDs are conditions commonly characterised by the presence of pain or decreased function. ${ }^{9}$ Therefore, workplace physical activity interventions often focus on numerous outcomes related to the individual such as pain, discomfort or fatigue, ${ }^{10-17}$ physical function such 
as strength, flexibility or endurance ${ }^{8}$ and psychosocial function such as quality of life, job satisfaction or wellbeing. ${ }^{18} 19$ In theory, the workplace environment does offer the possibility to reach and to raise awareness of a large number of workers. ${ }^{20}$ However, workplace physical activity programmes are less often offered and performed by those at risk of developing WMSDs (ie, low status, low income and blue-collar workers). ${ }^{721}{ }^{22}$ Furthermore, a $40 \%-60 \%$ compliance is commonly observed whatever the duration of the programmes. ${ }^{23-28}$ It is presumable that these observations could partly stem from 'practical' barriers to offer physical activity at the workplace, such as time constraints, time of the day and duration of the training sessions. ${ }^{29-33}$ In other words, programmes should be easy to implement in the daily routine of the employees as well as of the employers. This application recommendation is supported by scientific results that shown that short bouts of exercises are easier to fit in organisational routines than long sessions. ${ }^{34}{ }^{35}$ For instance, Andersen $e t$ $a b^{34}$ in a 10 -week workplace physical activity programme among office-workers, have compared the effects of a same weekly training volume, that is, 1 hour performed with different training frequencies (from one session per week to nine sessions per week) on training adherence. These authors have reported that adherence among office workers was significantly higher when the training volume was divided at least into 3 weekly training sessions.

In the last few years, the implementation of physical warm-up prior the beginning of the working days is increasingly adopted in companies to manage WMSDs (INRS 2018). In these companies, it is common to observe warm-up lasting between 5 and $15 \mathrm{~min}$ a day as well supervised by professionals such as sport trainer or physiotherapist as trained employees. ${ }^{36}$ Previous reviews have found positive effects of warm-up on performance ${ }^{37}$ and injury prevention in sports. ${ }^{37-41}$ However, it is surprising that data on the effects of warm-up on WMSD are scarce and, when available, lead to rather conflicting/inconclusive results. ${ }^{42-44}$ Within this context, the aim of this systematic review will be to evaluate the effectiveness of workplace warm-up interventions on WMSDs and physical and psychosocial functions among workers.

\section{METHODS}

The present review protocol is being reported in accordance with the reporting guidance the Preferred Reporting Items for Systematic Reviews and Meta-Analyses Protocols (PRISMA-P) statement ${ }^{45}$ (see PRISMA-P checklist, online supplemental additional file 1 ). This review will be reported in accordance with the PRISMA statement.

\section{Criteria for considering studies for this review}

\section{Type of studies}

Original quantitative research studies that assessed the effect of a warm-up intervention in a workplace setting aiming at preventing WMSDs or musculoskeletal pain or discomfort or fatigue in the worker will be included in the review.

As correctly argued, randomised controlled trials (RCTs) are considered as the gold standard to assess the effectiveness of an intervention. ${ }^{46}$ However, its implementation in occupational setting may not always be feasible and its implementation is called into question. ${ }^{17-51}$ In that specific case, recent studies have suggested that non-RCT may maximise the body of evidence and have suggested including non-RCT in systematic-reviews. ${ }^{52-54}$ For these reasons and as previously done in recent systematic reviews covering the scope of the present review, ${ }^{10} 49$ both randomised and non-randomised controlled studies will be included. Therefore, quasi-RCTs (participants not randomly allocated), cluster randomised trials (ie, randomisation of a group of people, eg, randomisation at a company level), preference trials (patients can choose their treatment) and before-and-after study are design which will be included.

Period of studies publication was defined from inception onwards to June 2020. Finally, to be eligible for inclusion, studies had to be published in English in peerreviewed scientific journals. ${ }^{19} 49$ As only studies in English will be included and may lead to reporting bias, we will report potentially eligible studies in other languages.

The following types of studies will be ineligible: case reports, abstracts, editorials, conference abstracts, letters to the editor, reviews and meta-analysis. Studies will be also excluded if the intervention was partially or totally implemented outside of the workplace, for example, in a clinical setting and if the intervention was implemented in combination with another intervention, for example, ergonomics. Therefore, studies will be excluded when differences can not only be attributed only to the warm-up intervention.

\section{Types of participants}

This review will include adult employees (18 years of age or older) and will exclude adults with specific comorbidities or diseases (such as diabetes, arthritis, cancer, stroke) and/or special populations (pregnant, severe or rare physical disability or cognitive disability).

\section{Types of intervention}

This review will include studies which have implemented warm-up interventions in real workplaces. To facilitate the comprehension of a warm-up intervention, we will use the definition given by McCrary et $a l^{37}$ that is, 'a warm-up is a protocol specifically undertaken to prepare the onset of subsequent physical activity', in our case a working activity.

As recently used in a systematic review by Luger $e t a l^{\tilde{5}}$ to describe work-break programmes and a study by Slade and Keating ${ }^{56}$ about exercise prescription, we will characterise the warm-up intervention with the following four components:

1. Duration: warm-up may last from 5 min to 1 hour.

2. Frequency: warm-up may differ in number. 
3. Type: warm-up may be stretching as well as cardiotraining exercises or combination of strengthening exercises.

4. Intensity: warm-up may be performed with/without load or performed at a low or high percentage of the maximum heart rate.

Studies will be excluded from this review if the warm-up intervention was partially or totally implemented outside of the workplace, for example, in a clinical setting or under laboratory conditions and if the warm-up intervention was implemented in combination with another intervention, for example, ergonomics.

\section{Comparator}

Inclusion criteria: We will consider studies that compared the warm-up intervention with a non-treatment control group (eg, no intervention or usual activity or another type of workplace physical activity) or a non-active comparator (eg, leaflets on benefits of physical activity).

Exclusion criteria: Studies with no comparison measures.

\section{Types of outcome measures}

\section{Main outcomes}

WMSDs are defined as a group of conditions or health problems affecting the locomotor apparatus. These conditions are characterised by pain, impaired function, overall fatigue and stress. ${ }^{9}{ }^{57}$ Therefore, among primary outcomes we will include all the outcomes associated with work related musculoskeletal issues that are (1) participant's musculoskeletal pain through the use of pain scales (eg, Numeric Rating Scale or Visual Analogue Scale) or questionnaire (eg, McGill pain questionnaire) ${ }^{55}$ and (2) participant discomfort or fatigue ${ }^{105}$ through validated scales and (3) physical function as measured or estimated by questionnaires, scales, performances and/or specific tests. Dichotomous data such as presence/absence of symptoms will be also considered.

\section{Secondary outcomes}

For the prevention of the consequences of WMSDs we will include-if possible-and as secondary outcomes, all the outcomes associated with psychosocial function such as the measure of quality of life, job satisfaction, job control or motivation at work. In this review job control is considered as an indicator of psychosocial stress at work. ${ }^{58}$ This indicator is often measured with the job demand-control support model developed by Karasek et al..$^{59}$

\section{Information sources and search strategy}

Four electronic databases-Cochrane Central Register of Controlled Trials, PubMed (Medline), Web of Science and Physiotherapy Evidence Database-will be searched systematically from inception onwards to identify studies satisfying the search criteria. Note that these databases have previously used in published reviews covering the scope of this review. ${ }^{4955601}$ The proposed search strategy terms for Medline are listed in table 1 and will be modified to fit the index system of other databases.
Table 1 Sample MEDLINE search strategy terms (Mesh: Mesh terms ; ti: tittle ; ab: abstract)

\begin{tabular}{|c|c|}
\hline & Keywords \\
\hline 1 & Workplace(Mesh) \\
\hline 2 & Work $^{\star}$ ti,ab \\
\hline 3 & Employ ti,ab \\
\hline 4 & Compan ${ }^{\star}$ ti,ab \\
\hline 5 & 1 OR 2 OR 3 OR 4 \\
\hline 6 & Warm-Up Execise(Mesh) \\
\hline 7 & Pre-shift ti,ab \\
\hline 8 & Pre-exercise ${ }^{\star}$ ti,ab \\
\hline 9 & Pre-activit* ti,ab \\
\hline 10 & 6 OR 7 OR 8 OR 9 \\
\hline 11 & Musculoskeletal diseases(Mesh) \\
\hline 12 & Pain(Mesh) \\
\hline 13 & Musculoskeletal Pain(Mesh) \\
\hline 14 & WMSD* ti,ab \\
\hline 15 & Pain ti,ab \\
\hline 16 & (endurance or strength or flexibility) ti,ab \\
\hline 17 & $\begin{array}{l}\text { (quality of life or job satisfaction or work ability or well- } \\
\text { being or stress or disabilit* or health or discomfort or } \\
\text { comfort or fatigue or injur*) ti,ab }\end{array}$ \\
\hline \multirow[t]{2}{*}{18} & 11 OR 12 OR 13 OR 14 OR 15 OR 16 OR 17 \\
\hline & Combining search terms \\
\hline 20 & 5 AND 10 AND 18 \\
\hline
\end{tabular}

\section{Additional intended information sources}

To be sure not to miss relevant studies for the review, the reference list of for all eligible articles will be checked. Then, a grey literature search will be performed on ClinicalTrials.gov. Finally, experts in this domain will be contacted to collect information on unknown or ongoing studies.

\section{Data collection}

Study selection process

All studies that met inclusion criteria passed through a data extraction and quality assessment process performed by two independent reviewers. A third reviewer will be requested to resolve disagreement when consensus could not be reached. Reviewers will not be blinded to study author(s) or journal title. At stage 1, two independent reviewers will screen abstract and titles identified from the search strategy. At stage 2, the same two reviewers will screen the full-text articles for inclusion. At this stage, all reasons for exclusion of articles will be recorded and reported. Finally, the relevant studies, which respect eligibility criteria, will be screened by a senior review team member $(\mathrm{NV})$ to be included in the systematic review.

\section{Data extraction and management}

First a data extraction form will be created and validated by the three team members. This data collection form will 
be fulfilled by one team member (NL) and corrected by another team member (RB). Any disagreement between the two reviewers will be resolved by consensus or discussion with the senior review team member $(\mathrm{NV})$. This extraction form could be modified from the information collected in the eligible studies but should at least specify the following information ${ }^{606} 63$ :

- General: authors, year of publication, journal's name, source of funding (if any) and country of the study.

- Methods: study design, total duration of study, follow-up when data were collected, study setting and withdrawals.

- Participants: number, age, gender, inclusion/exclusion criteria, type of workplace or job task, health of the workers/health status, that is, asymptomatic or symptomatic, year of work experience.

- Interventions: description of the type, duration, frequency, intensity, supervision of the warm-up programme, description/content of the comparison/ control group and number of participants allocated to each group.

- Data collection: primary and secondary outcomes, measurement tools, questionnaires, tests.

- Statistical tests.

- Main results.

\section{Risk of bias (quality) assessment}

Two team members (NL and $\mathrm{RB}$ ) will independently assess the risk of bias for each included study. Any disagreement between team members will be solved by consensus or discussion with the third team member. As both randomised and non-randomised controlled studies will be included in this review, two risk of bias tools will be used.

\section{For RCT}

The Cochrane tool for assessing risk of bias from the Cochrane Handbook for Systematic Reviews of Interventions will be used to assess potential biases of the included studies. This tool is a well-known and validated instrument to assess the risk of bias in RCTs ${ }^{64}$ This tool has been revised in $2019^{65}$ and has now five domains to assess bias arising from: (1) randomisation process, (2) deviation from the intended intervention, (3) missing outcome data, (4) measurement of the outcome and (5) selection of the reported result. Each domain will be scored as follow (see table 2): 'high risk of bias', 'low risk of bias' and 'some concerns'.

\section{For non-RCT}

The Risk Of Bias In Non-randomised Studies-of Interventions (ROBINS-I) will be used to asses potential biases of the included non-RCT. ${ }^{66}$ This tool has seven domains to assess bias arising from (1) confounding, (2) selection of participants, (3) classification of the intervention, (4) deviations from the intended intervention, (5) missing data, (6) measurement of outcomes and (7) selection of the reported result.
Table 2 Risk of bias judgement for a specific domain (from Sterne et $a /^{65}$ )

Overall risk of

bias judgement Criteria

Low risk of bias The study is judged to be at low risk of bias for all domains for this result

Some concerns The study is judged to be at high risk of bias in at least one domain for this result, but not to be at high risk of bias for any domain.

High risk of bias The study is judged to be at high risk of bias in at least one domain for this result Or

The study is judged to have some concerns for multiple domains in a way that substantially lowers confidence in the result.

\section{Measures of treatment effect}

For studies using continuous data, treatment effect will be reported as mean difference with $95 \%$ CI. In case the studies evaluate the same outcome with different scales, standardised mean difference with $95 \%$ CI will be calculated. Regarding dichotomous/categorical variables, the treatment effect will be calculated using the relative risk (RR) with $95 \%$ CI. ${ }^{67-70}$ Since the number of included studies is greater than $5^{68}$ and when these studies are considered as sufficiently homogeneous, outcome data will be synthesised using a random effect meta-analysis. ${ }^{55} 697172$ If meta-analysis is not possible due to heterogeneity or if the outcomes cannot be pooled, a narrative synthesis will be performed using text and table formats. Results will be also presented in forest plots.

\section{Assessment of statistical heterogeneity}

Statistical heterogeneity, defined as variability in the intervention effects will be estimated using the $\chi^{2}$ test, with $\chi^{2}$ $\mathrm{p}>0.10$ provides significant evidence of heterogeneity. $\chi^{2}$ assesses whether heterogeneity is only due to chance. To ensure a right comprehension of heterogeneity, $\chi^{2}$ will be completed with $\mathrm{I}^{2}$ statistics particularly relevant when studies have small sample size or are few in numbers. Heterogeneity will be categorised as follows ${ }^{72}$ :

- 0\%-40\%: not be important.

- 30\%-60\%: moderate heterogeneity.

- 50\%-90\% substantial heterogeneity.

- 75\%-100\%: considerable heterogeneity.

\section{Quality assessment and strategy for data synthesis}

To assess quality of evidence of the included studies the Grading of Recommendations, Assessment, Development and Evaluation (GRADE) approach ${ }^{73}$ will be used. This approach grades studies as followed: very low, low, moderate and high. As suggested by Bordado $e t a l^{19}$ the quality assessment will be based on the findings in data extraction, and will follow the domains of quality evaluation in the GRADE approach: risk of bias, inconsistency, 
indirectness and imprecision. Two team members (NL and $\mathrm{RB}$ ) will independently assess the quality of evidence of the included studies with the GRADE approach.

\section{Analysis of subgroups or subsets}

In case a sufficient number of studies are included in the review, a subgroup analysis will be performed. The latter will be carried out for each outcome and for the following factors: (1) participants' characteristics (eg, sex, age. If possible authors will compare participants aged 40 years and younger with participants aged 41 years and older), (2) WMSDs location (eg, neck vs low back vs upper extremities), (3) occupational activity (eg, active vs sedentary jobs), (4) length of intervention, (5) study design (eg, RCT vs non-RCT) and (6) comparison group type (eg, passive vs active control group $)^{5570}$ and (7) implementation warm-up intervention (supervised vs non supervised). ${ }^{74}$

\section{Sensitivity analysis}

The authors of the present systematic review planned to perform sensitivity analysis to determine whether our findings are affected by high risk of bias and baseline pain. They also planned to combine the outcomes concerning pain, discomfort or fatigue and physical function. To perform sensitivity analysis, studies will be considered to be at high risk of bias if one of the main biases would be rated unclear or high risk (ie, random sequence allocation, allocation concealment, incomplete outcome data or selective outcome reporting. ${ }^{63}$ Concerning pain, the low-intensity pain threshold was defined as 3 out of 10 on a pain intensity scale. ${ }^{756}$

\section{ETHICS AND DISSEMINATION}

No ethic committee was required to conduct this review. However, all included studies in this review will follow current ethical standard and will be in accordance with the Declaration of Helsinki. The results of this review will be submitted for publication to a peer-reviewed high-impact academic journal. Other dissemination may include presentations at international conferences, seminars and note to social media to influence decision-makers.

\section{DISCUSSION}

Workplace physical activity is now well recognised as a potential intervention to prevent WMSDs. ${ }^{7811-17}$ Although benefits of a warm-up have been previously documented in sports context, ${ }^{37-41}$ to the best of our knowledge, the effectiveness of such intervention in workplaces remains to be established. Interestingly, the primary outcome analysed in this review will be associated with WMSDs such as pain, discomfort or fatigue. The secondary outcomes will be related to physical or psychosocial functions. All these outcomes recognised to be decreased in case of WMSDs are also the main outcomes reported in studies assessing the effects on an intervention on WMSDs. ${ }^{10-16}$ For these reasons, these findings could constitute a solid starting point to help clinicians, researchers, companies and policy-makers trying to reduce the burden of WMSDs.

\section{LIMITATIONS AND STRENGTHS}

Our review presents several strengths. The major strength is the systematic procedure employed. In this sense, a large number of scientific databases will be searched. Then, two reviewers will independently screen articles, rate the quality of these studies and the risk of bias. Finally, the use of recommended standard reporting instruments such as PRISMA-P, ROBINS-I and GRADE will strengthen the recommendations that should be made at the end of the review. At this point, however, we are aware that the potential strength of this review could be reduced by the lack of high-quality trials and high heterogeneity. First, the recent scientific literature confirms that RCT in a workplace context are, of course possible but rare. ${ }^{3477-79}$ In this sense, numerous authors have concluded that considerable efforts had to be made to overcome difficulties to implement such study design, but also to recruit a large number of employees. ${ }^{14-48} 80$ To deal with this heterogeneity, the authors have preplanned to perform a subgroup and a sensitivity analysis. This choice will allow knowing whether or not the intervention effects differ between trials. Then, we are also aware that including both RCT and non-RCT will therefore lead to downgrade the validity and strength of the review and will increase the risk of bias especially for the blinding and generation domains. ${ }^{70}$ Second, a recent review of literature by Johnson $e t a l^{81}$ on how outcomes are measured in workplace physical activity interventions have reported heterogeneous measurement tools and data collection making comparisons between studies rather difficult. To conclude, although the researchers do not anticipate protocol amendments, issues that arise with the original protocol will be documented in the review paper under the methodology section.

Contributors All listed authors have contributed and will continue to contribute meaningfully to the protocol and proposed review. NL, RB and NV conceived the proposed review and developed the search strategy. NL and RB are the two title and abstract reviewers, and NL and RB arethe two full-text reviewers. NV will be the third reviewer that will help resolve any discrepancy. RB submitted the protocol to PROSPERO and is responsible for updating the registered protocol as needed. All authors read the final protocol manuscript and revised it forcontent; all also approved the final version.

Funding This review is part of a PhD thesis-project conducted in the University of Grenoble Alpes and Opti'Mouv. The research project is promoted by the University of Grenoble Alpes and partially financed by the 'Ministère de l'Enseignement Supérieur et de la Recherche' via the 'Association Nationale Recherche Technologie' (ANRT) by means of the 'Convention Industrielle de Formation par la Recherche' (CIFRE) grant (n 2019/0488). The founding source has no role in the study design, data collection, results interpretation or manuscript writing.

Competing interests Opti'Mouv is a company that provides workplace health promotion services as workplace physical activity programmes.

Patient consent for publication Not required.

Provenance and peer review Not commissioned; externally peer reviewed.

Supplemental material This content has been supplied by the author(s). It has not been vetted by BMJ Publishing Group Limited (BMJ) and may not have been 
peer-reviewed. Any opinions or recommendations discussed are solely those of the author(s) and are not endorsed by BMJ. BMJ disclaims all liability and responsibility arising from any reliance placed on the content. Where the content includes any translated material, BMJ does not warrant the accuracy and reliability of the translations (including but not limited to local regulations, clinical guidelines, terminology, drug names and drug dosages), and is not responsible for any error and/or omissions arising from translation and adaptation or otherwise.

Open access This is an open access article distributed in accordance with the Creative Commons Attribution Non Commercial (CC BY-NC 4.0) license, which permits others to distribute, remix, adapt, build upon this work non-commercially, and license their derivative works on different terms, provided the original work is properly cited, appropriate credit is given, any changes made indicated, and the use is non-commercial. See: http://creativecommons.org/licenses/by-nc/4.0/.

\section{ORCID iD}

Romain Balaguier http://orcid.org/0000-0001-6393-1556

\section{REFERENCES}

1 Punnett L. Musculoskeletal disorders and occupational exposures: how should we judge the evidence concerning the causal association? Scand J Public Health 2014;42:49-58.

2 Bayattork M, Jakobsen MD, Sundstrup E, et al. Musculoskeletal pain in multiple body sites and work ability in the general working population: cross-sectional study among 10,000 wage earners. Scand J Pain 2019;19:131-7.

3 Bevan S. Economic impact of musculoskeletal disorders (MSDs) on work in Europe. Best Pract Res Clin Rheumatol 2015;29:356-73.

4 Woolf AD, Erwin J, March L. The need to address the burden of musculoskeletal conditions. Best Pract Res Clin Rheumatol 2012;26:183-224.

5 Blyth FM, Briggs AM, Schneider $\mathrm{CH}$, et al. The global burden of musculoskeletal pain - where to from here?. Am J Public Health 2019;109:35-40.

6 James SL, Abate D, Abate KH, et al. Global, regional, and national incidence, prevalence, and years lived with disability for 354 diseases and injuries for 195 countries and territories, 1990-2017: a systematic analysis for the global burden of disease study 2017. Lancet 2018;392:1789-858.

7 Holtermann A, Mathiassen SE, Straker L. Promoting health and physical capacity during productive work: the Goldilocks principle. Scand J Work Environ Health 2019;45:90-7.

8 Siøgaard G, Christensen JR, Justesen JB, et al. Exercise is more than medicine: the working age population's well-being and productivity. J Sport Health Sci 2016:5:159-65.

9 Søgaard K, Sjøgaard G. Physical activity as cause and cure of muscular pain: evidence of underlying mechanisms. Exerc Sport Sci Rev 2017:45:136-45.

10 Hoosain M, de Klerk S, Burger M. Workplace-based rehabilitation of upper limb conditions: a systematic review. J Occup Rehabil 2019:29:175-93.

11 Chen X, Coombes BK, Sjøgaard G, et al. Workplace-based interventions for neck pain in office workers: systematic review and meta-analysis. Phys Ther 2018;98:40-62.

12 Coury HJCG, Moreira RFC, Dias NB. Evaluation of the effectiveness of workplace exercise in controlling neck, shoulder and low back pain: a systematic review. Braz J Phys Ther 2009;13:461-79.

13 Moreira-Silva I, Teixeira PM, Santos R, et al. The effects of workplace physical activity programs on musculoskeletal pain: a systematic review and meta-analysis. Workplace Health Saf 2016;64:210-22.

14 Rodrigues EV, Gomes ARS, Tanhoffer AIP, et al. Effects of exercise on pain of musculoskeletal disorders: a systematic review. Acta ortop. bras. 2014;22:334-8.

15 Skamagki G, King A, Duncan M, et al. A systematic review on workplace interventions to manage chronic musculoskeletal conditions. Physiother Res Int 2018;23:e1738.

16 Proper KI, van Oostrom SH. The effectiveness of workplace health promotion interventions on physical and mental health outcomes - a systematic review of reviews. Scand J Work Environ Health 2019:45:546-59.

17 Van Eerd D, Munhall C, Irvin E, et al. Effectiveness of workplace interventions in the prevention of upper extremity musculoskeletal disorders and symptoms: an update of the evidence. Occup Environ Med 2016;73:62-70.

18 Abdin S, Welch RK, Byron-Daniel J, et al. The effectiveness of physical activity interventions in improving well-being across office-based workplace settings: a systematic review. Public Health 2018;160:70-6.

19 Bordado Sköld M, Bayattork M, Andersen LL, et al. Psychosocial effects of workplace exercise - A systematic review. Scand J Work Environ Health 2019;45:533-45.

20 Kuoppala J, Lamminpää A, Husman P. Work health promotion, job well-being, and sickness absences--a systematic review and metaanalysis. J Occup Environ Med 2008;50:1216-27.

21 Jørgensen MB, Villadsen E, Burr H, et al. Does workplace health promotion in Denmark reach relevant target groups? Health Promot Int 2015;30:318-27.

22 Macniven R, Engelen L, Kacen MJ, et al. Does a corporate worksite physical activity program reach those who are inactive? findings from an evaluation of the global corporate challenge. Health Promot $J$ Austr 2015;26:142-5.

23 Andersen $\mathrm{CH}$, Andersen LL, Zebis MK, et al. Effect of scapular function training on chronic pain in the neck/shoulder region: a randomized controlled trial. J Occup Rehabil 2014;24:316-24.

24 Hagberg M, Harms-Ringdahl K, Nisell R, et al. Rehabilitation of neck-shoulder pain in women industrial workers: a randomized trial comparing isometric shoulder endurance training with isometric shoulder strength training. Arch Phys Med Rehabil 2000;81:1051-8.

25 Jakobsen MD, Sundstrup E, Brandt M, et al. Factors affecting pain relief in response to physical exercise interventions among healthcare workers. Scand J Med Sci Sports 2017;27:1854-63.

26 Jay K, Frisch D, Hansen K, et al. Kettlebell training for musculoskeletal and cardiovascular health: a randomized controlled trial. Scand J Work Environ Health 2011;37:196-203.

27 Jay K, Brandt M, Hansen K, et al. Effect of individually tailored biopsychosocial workplace interventions on chronic musculoskeletal pain and stress among laboratory technicians: randomized controlled trial. Pain Physician 2015;18:459-71.

28 Viljanen M, Malmivaara A, Uitti J, et al. Effectiveness of dynamic muscle training, relaxation training, or ordinary activity for chronic neck pain: randomised controlled trial. BMJ 2003;327:475.

29 Andersen LL, Zebis MK. Process evaluation of workplace interventions with physical exercise to reduce musculoskeletal disorders. Int J Rheumatol 2014;2014:1-11.

30 Bredahl TVG, Særvoll CA, Kirkelund L, et al. When intervention meets organisation, a qualitative study of motivation and barriers to physical exercise at the workplace. Sci World J 2015;2015:518561

31 Chau J, Engelen L, Kolbe-Alexander T, et al. 'In initiative overload': Australian perspectives on promoting physical activity in the workplace from diverse industries. Int J Environ Res Public Health 2019;16:516

32 Planchard J-H, Corrion K, Lehmann L, et al. Worksite physical activity barriers and facilitators: a qualitative study based on the Transtheoretical model of change. Front Public Health 2018;6:326.

33 Wierenga D, Engbers LH, Van Empelen P, et al. What is actually measured in process evaluations for worksite health promotion programs: a systematic review. BMC Public Health 2013;13:1190.

34 Andersen $\mathrm{CH}$, Andersen LL, Gram B, et al. Influence of frequency and duration of strength training for effective management of neck and shoulder pain: a randomised controlled trial. Br J Sports Med 2012;46:1004-10.

35 Dalager T, Bredahl TGV, Pedersen MT, et al. Does training frequency and supervision affect compliance, performance and muscular health? a cluster randomized controlled trial. Man Ther 2015;20:657-65.

36 Balaguier R, Madeleine P, Rose-Dulcina K, et al. Effects of a worksite supervised adapted physical activity program on trunk muscle endurance, flexibility, and pain sensitivity among vineyard workers. $J$ Agromedicine 2017;22:200-14.

37 McCrary JM, Ackermann BJ, Halaki M. A systematic review of the effects of upper body warm-up on performance and injury. Br J Sports Med 2015;49:935-42.

38 Fradkin AJ, Zazryn TR, Smoliga JM. Effects of warming-up on physical performance: a systematic review with meta-analysis. $J$ Strength Cond Res 2010;24:140-8.

39 Hammami A, Zois J, Slimani M, et al. The efficacy and characteristics of warm-up and re-warm-up practices in soccer players: a systematic review. J Sports Med Phys Fitness 2018;58:135-49.

40 Neiva HP, Marques MC, Barbosa TM, et al. Warm-up and performance in competitive swimming. Sports Med 2014;44:319-30.

41 Silva LM, Neiva HP, Marques MC, et al. Effects of warm-up, PostWarm-Up, and Re-Warm-Up strategies on explosive efforts in team sports: a systematic review. Sports Med 2018;48:2285-99.

42 Aje OO, Smith-Campbell B, Bett C. Preventing musculoskeletal disorders in factory workers: evaluating a new eight minute stretching program. Workplace Health Saf 2018;66:343-7. 
43 Gartley RM, Prosser JL. Stretching to prevent musculoskeletal injuries. An approach to workplace wellness. Aaohn J 2011;59:247-52.

44 Holmström E, Ahlborg B. Morning warming-up exercise--effects on musculoskeletal fitness in construction workers. Appl Ergon 2005;36:513-9.

45 Moher D, Shamseer L, Clarke M, et al. Preferred reporting items for systematic review and meta-analysis protocols (PRISMA-P) 2015 statement. Syst Rev 2015;4:1.

46 Burdorf A, van der Beek AJ. To RCT or not to RCT: evidence on effectiveness of return-to-work interventions. Scand $J$ Work Environ Health 2016;42:257-9.

47 Burton J. WHO healthy workplace framework and model: background and supporting literature and practices, 2010.

48 Kwak L, Kremers SPJ, van Baak MA, et al. Participation rates in worksite-based intervention studies: health promotion context as a crucial quality criterion. Health Promot Int 2006;21:66-9.

49 Malik SH, Blake H, Suggs LS. A systematic review of workplace health promotion interventions for increasing physical activity. $\mathrm{Br} J$ Health Psychol 2014;19:149-80.

50 Marshall AL. Challenges and opportunities for promoting physical activity in the workplace. J Sci Med Sport 2004;7:60-6.

51 Schlevis RMC, Oude Hengel KM, Burdorf A, et al. Evaluation of occupational health interventions using a randomized controlled trial: challenges and alternative research designs. Scand J Work Environ Health 2015;41:491-503.

52 Cuello-Garcia CA, Morgan RL, Brozek J, et al. A scoping review and survey provides the rationale, perceptions, and preferences for the integration of randomized and nonrandomized studies in evidence syntheses and grade assessments. J Clin Epidemiol 2018;98:33-40.

53 Reeves BC, Higgins JPT, Ramsay C, et al. An introduction to methodological issues when including non-randomised studies in systematic reviews on the effects of interventions. Res Synth Methods 2013;4:1-11.

54 Schünemann HJ, Tugwell P, Reeves BC, et al. Non-randomized studies as a source of complementary, sequential or replacement evidence for randomized controlled trials in systematic reviews on the effects of interventions. Res Synth Methods 2013;4:49-62.

55 Luger T, Maher CG, Rieger MA, et al. Work-break schedules for preventing musculoskeletal disorders in workers. Cochrane Database Syst Rev 2017;2017.

56 Slade SC, Keating JL. Exercise prescription: a case for standardised reporting. Br J Sports Med 2012;46:1110-3.

57 Office for Official Publ. of the Europ. Communities. OSH in figures: work-related musculoskeletal disorders in the EU - Facts and figures, 2010.

58 Too LS, Leach L, Butterworth P. Is the association between poor job control and common mental disorder explained by general perceptions of control? findings from an Australian longitudinal cohort. Scand J Work Environ Health 2020;46:311-20.

59 Karasek R, Brisson C, Kawakami N, et al. The Job Content Questionnaire (JCQ): an instrument for internationally comparative assessments of psychosocial job characteristics. J Occup Health Psychol 1998;3:322-55.

60 Coenen P, Huysmans MA, Holtermann A, et al. Do highly physically active workers die early? A systematic review with meta-analysis of data from 193696 participants. Br J Sports Med 2018;52:1320-6.

61 Sultan-Taïeb H, Parent-Lamarche A, Gaillard A, et al. Economic evaluations of ergonomic interventions preventing work-related musculoskeletal disorders: a systematic review of organizationallevel interventions. BMC Public Health 2017;17:935.

62 Padula RS, Comper MLC, Sparer EH, et al. Job rotation designed to prevent musculoskeletal disorders and control risk in manufacturing industries: a systematic review. Appl Ergon 2017;58:386-97.
63 Luger T, Maher CG, Rieger MA, et al. Work-break schedules for preventing musculoskeletal symptoms and disorders in healthy workers. Cochrane Database Syst Rev 2019;7:CD012886.

64 Higgins JPT, Altman DG, Gøtzsche PC, et al. The Cochrane collaboration's tool for assessing risk of bias in randomised trials. BMJ 2011;343:d5928

65 Sterne JAC, Savović J, Page MJ, et al. Rob 2: a revised tool for assessing risk of bias in randomised trials. BMJ 2019;366:14898.

66 Sterne JA, Hernán MA, Reeves BC, et al. ROBINS-I: a tool for assessing risk of bias in non-randomised studies of interventions. BMJ 2016;355:i4919.

67 Dos Santos Franco YR, Miyamoto GC, Franco KFM, et al. Exercise therapy in the treatment of tendinopathies of the lower limbs: a protocol of a systematic review. Syst Rev 2019;8:142.

68 Huffman MK, Reed JB, Carpenter T, et al. Maintenance motives for physical activity among older adults: a protocol for a systematic review and meta-analysis. BMJ Open 2020;10:e032605

69 Larsen RT, Christensen J, Juhl CB, et al. Physical activity monitors to enhance the daily amount of physical activity in elderly-a protocol for a systematic review and meta-analysis. Syst Rev 2018;7:69.

70 Seeberg KGV, Andersen LL, Bengtsen E, et al. Effectiveness of workplace interventions in rehabilitating musculoskeletal disorders and preventing its consequences among workers with physical and sedentary employment: systematic review protocol. Syst Rev 2019;8:219.

71 Ubago-Guisado E, Gracia-Marco L, Cavero-Redondo I, et al. Effect of different types of exercise on health-related quality of life during and after cancer treatment: a protocol for a systematic review and network meta-analysis. BMJ Open 2019;9:e031374.

72 Jones RA, Lawlor ER, Griffin SJ, et al. Impact of adult weight management interventions on mental health: a systematic review and meta-analysis protocol. BMJ Open 2020;10:e031857.

73 Guyatt G, Oxman AD, Akl EA, et al. Grade guidelines: 1. IntroductionGRADE evidence profiles and summary of findings tables. J Clin Epidemiol 2011;64:383-94.

74 Matarán-Peñarrocha GA, Lara Palomo IC, Antequera Soler E, et al. Comparison of efficacy of a supervised versus non-supervised physical therapy exercise program on the pain, functionality and quality of life of patients with non-specific chronic low-back pain: a randomized controlled trial. Clin Rehabil 2020;34:948-59.

75 Moore RA, Straube S, Aldington D. Pain measures and cut-offs - 'no worse than mild pain' as a simple, universal outcome. Anaesthesia 2013;68:400-12.

76 Parry SP, Coenen P, Shrestha N, et al. Workplace interventions for increasing standing or walking for decreasing musculoskeletal symptoms in sedentary workers. Cochrane Database Syst Rev 2019;2019

77 Jakobsen MD, Sundstrup E, Brandt M, et al. Effect of workplaceversus home-based physical exercise on musculoskeletal pain among healthcare workers: a cluster randomized controlled trial. Scand J Work Environ Health 2015;41:153-63.

78 Jørgensen MB, Rasmussen CDN, Ekner D, et al. Successful reach and adoption of a workplace health promotion RCT targeting a group of high-risk workers. BMC Med Res Methodol 2010;10:56

79 Andersen LL, Saervoll CA, Mortensen OS, et al. Effectiveness of small daily amounts of progressive resistance training for frequent neck/shoulder pain: randomised controlled trial. Pain 2011;152:440-6.

80 Schelvis RMC, Oude Hengel KM, Burdorf A, et al. Evaluation of occupational health interventions using a randomized controlled trial: challenges and alternative research designs. Scand J Work Environ Health 2015;41:491-503.

81 Johnson S, Regnaux J-P, Marck A, et al. Understanding how outcomes are measured in workplace physical activity interventions: a scoping review. BMC Public Health 2018;18:1064. 